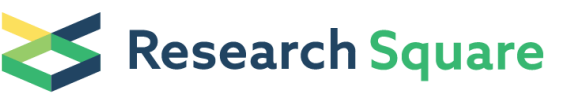 \\ Preprints are preliminary reports that have not undergone peer review. \\ They should not be considered conclusive, used to inform clinical practice, or referenced by the media as validated information.
}

\section{Structural, Magnetic, and Magnetocaloric Properties of R2NiMnO6 (R = Eu, Gd, Tb)}

\section{K.P. Shinde}

Department of Materials Science and Engineering, Hanbat National University, Daejeon 34158, South Korea

\section{E.J. Lee}

Department of Materials Science and Engineering, Hanbat National University, Daejeon 34158, South Korea

\section{Maykel Manawan}

Fakultas Teknologi Pertahanan, Universitas Pertahanan Indonesia, Bogor 16810, Indonesia

\section{A. Lee}

Center for Scientific Instrumentation, Korea Basic Science Institute, Daejeon 34133, South Korea

\section{S.Y. Park}

Center for Scientific Instrumentation, Korea Basic Science Institute, Daejeon 34133, South Korea

\section{Younghun Jo}

Center for Scientific Instrumentation, Korea Basic Science Institute, Daejeon 34133, South Korea

\section{$\mathrm{K} \mathrm{Ku}$}

Department of Materials Science and Engineering, Hanbat National University, Daejeon 34158, South Korea

\section{J.M. Kim}

Department of Materials Science and Engineering, Hanbat National University, Daejeon 34158, South Korea

\section{J.S.Park ( $\nabla$ jsphb@hanbat.ac.kr)}

Department of Materials Science and Engineering, Hanbat National University, Daejeon 34158, South Korea

\section{Research Article}

Keywords: Magnetocaloric Properties , R2NiMn06, Double perovskite, ceramic powder

Posted Date: July 15th, 2021

DOl: https://doi.org/10.21203/rs.3.rs-701309/v1

License: (c) (1) This work is licensed under a Creative Commons Attribution 4.0 International License. 


\section{Abstract}

Double perovskite $\mathrm{Eu}_{2} \mathrm{NiMnO}_{6}(\mathrm{ENMO}) \mathrm{Gd}_{2} \mathrm{NiMnO}_{6}(\mathrm{GNMO})$ and $\mathrm{Tb}_{2} \mathrm{NiMnO}_{6}(\mathrm{TNMO})$ ceramic powder have been synthesized by solid-state reaction and their crystal structure, microstructure, cryogenic magnetic properties, and magnetocaloric performance have been investigated. Structural studies by using X-ray diffraction shows that all compounds crystallize in the monoclinic structure with a $P 2_{1} / n$ space group. A ferromagnetic to paramagnetic (FM-PM) second-order phase transition occurred in ENMO, GNMO, and TNMO around 143, 130, and $112 \mathrm{~K}$, respectively. The values of maximum magnetic entropy change and relative cooling power at an applied field of $5 \mathrm{~T}$ are found to be $3.2,3.8,3.5 \mathrm{~J} / \mathrm{kgK}$ and 150 , $182,176 \mathrm{~J} / \mathrm{kg}$ respectively, for the studied sample. The change in structural, magnetic, and magnetocaloric effect ascribed to the superexchange mechanism of $\mathrm{Ni}^{2+}-\mathrm{O}-\mathrm{Mn}^{3+}$ and $\mathrm{Ni}^{2+}-\mathrm{O}-$ $\mathrm{Mn}^{4+}$. Due to different atomic size of Eu, Gd, Tb changes the ratio of $\mathrm{Mn}^{4+} / \mathrm{Mn}^{3+}$ which is responsible for the variation of properties significantly in double perovskite.

\section{Introduction}

Magnetic refrigeration technology based on the magnetocaloric effect (MCE) of the magnetic materials has been attracted many research groups over the conventional gas refrigeration. It shows the several advantages such as high energy efficient, eco-friendly, and cost-effective ${ }^{1-4}$. The MCE is a phenomenon of the coupling effect between the magnetic moment alignment with application and removal of magnetic field, which is strongly associated with the magnetic phase transition. It is also described as a variation of the isothermal magnetic entropy change or an adiabatic temperature change take place when the magnetic materials is kept under the changing magnetic field ${ }^{5,6}$. The magnetic refrigerant materials which are having a large MCE near room temperature as well as the cryogenic temperature region at lower magnetic fields are on focus of researchers because of their application in magnetic refrigeration ${ }^{7}$. Since, oxides are easily synthesized on large scale by using the conventional methods with an easily introducing different magnetic elements as dopants and adjustable transition temperature by optimizing experimental conditions. It has been reported that the materials based on perovskite and double perovskite oxides are able to enhance the MCE and relative cooling power ${ }^{8-11}$.

Double perovskites (DPs) with the general formula $A_{2} B B^{\prime} O_{6}$ having very interesting chemical as well as physical properties, with a wide range of the applications, due to this many research groups have attracted in the past decade. It is reported that, the atomic combination is extremely flexible with changing different $A, B$, and $B^{\prime}$ elements, which gives an interesting property such as magnetoresistance ${ }^{12,13}$, ferroelectricity ${ }^{14}$, magneto-capacitance ${ }^{15}$, and magnetic refrigeration ${ }^{16-18}$. The magnetocaloric effect in $\mathrm{Ln}_{2}(\mathrm{Ni} / \mathrm{Co}) \mathrm{MnO}_{6}$ have been reported by Jia et al. ${ }^{19}$. It was reported that these DPs showing multiple magnetic phase transitions and Tc decreased with lower ionic radii of rare earth elements. The magnetic transition from ferromagnetic to paramagnetic state in the double perovskite is associated with the superexchange mechanism $\mathrm{Mn}^{4+}-\mathrm{O}-\mathrm{Ni}^{2+} / \mathrm{Co}^{2+}$. Balli et al. have studied the 
present a large refrigerant capacity around $300 \mathrm{~K}$ and compared with large MCE materials ${ }^{20.21} \cdot \mathrm{R}_{2} \mathrm{NiMnO}_{6}$ $(\mathrm{R}=\mathrm{Pr}, \mathrm{Nd}, \mathrm{Tb}, \mathrm{Ho}$ and $\mathrm{Y}$ ) have been studied and compared with the perovskite materials having similar curie temperature by Chakraborty et $a^{22}$. The comparative study of $\mathrm{Eu}_{2} \mathrm{NiMnO}_{6}$ and $\mathrm{Dy}_{2} \mathrm{NiMnO}_{6}$ carried out by Su et al. and found that $-\Delta \mathrm{S}_{\mathrm{M}}$ reaches to $4.0 \mathrm{~J} / \mathrm{kgK}$ and $5.2 \mathrm{~J} / \mathrm{kgK}$ with $\Delta \mathrm{H}=7 \mathrm{~T}^{23}$. In $\mathrm{Dy}_{2} \mathrm{NiMnO}_{6}$ compound it was seen that the evolution of Griffiths phase which is due to the spin-phonon coupling which occurs because of smaller ionic radii of rare earth elements. In double perovskites, the important aspect to determine structural and magnetic or magnetocaloric properties are cation ordering between $\mathrm{B}$ and $\mathrm{B}^{\prime}$ element as well as distortion in bonding due to different A-site elements, which is believed to be the origin of complementary results. The small distortion by defects and disorders possibly leads to redistribution of electron density, thereby significantly changing electronic properties, magnetic ordering and magnetocaloric properties. However, unfortunately, little studies have been devoted to quantitatively revealing the correlation among the structure of double perovskite in particular, $\mathrm{B}-\mathrm{O}-\mathrm{B}^{\prime}$ bonding, bond length, and superexchange mechanism and subsequent magnetocaloric properties in these double perovskite systems.

In this paper, we have investigated the effect of ionic size of the rare earth element in $\mathrm{R}_{2} \mathrm{NiMnO}_{6}(R=\mathrm{Eu}$, $\mathrm{Gd}, \mathrm{Tb}$ ) double perovskite on the structural, magnetic, and magnetocaloric properties at cryogenic temperature. The different size of rare earth elements affects the bond angle analyzed by Rietveld refinement and X-ray photoemission (XPS) study by revealing $\mathrm{Mn}^{+4} / \mathrm{Mn}^{3+}$ ionic distribution in the double perovskite. The magnetocaloric properties are revealed at cryogenic temperature to understand its use as magnetic refrigerant for cooling application.

\section{Results And Discussion}

The phase purity and crystal structure of the samples are characterized by using the Rietveld refinement of room temperature X-ray diffraction data. Figure $1(a, b)$ show the Rietveld refined XRD pattern and the polyhedral crystal structure of the ENMO, GNMO, and TNMO sample, respectively. These diffraction patterns shows that all the studied compositions were crystallized in the monoclinic crystal system with space group $P 2{ }_{1} / n$. The structural parameters are refined by Rietveld's profile fitting method by using the TOPAS program ${ }^{24}$. The refinement parameters such as lattice parameters, bond length, the goodness of fit $\left(\chi^{2}\right)$, and bond angle of the studied compounds given in Table 1. It is seen that cell volume and crystal density increases with an increase in an atomic number of rare earth elements but the volume decreases. The crystal structure of GNMO is heavily tilted $\left(\beta=124^{\circ}\right)$, as compared to ENMO and TNMO $\left(\beta=\sim 90^{\circ}\right)$. 
Table 1

Structural parameters for ENMO, GNMO, and TNMO from the Rietveld refinement.

\begin{tabular}{|lllll|}
\hline & & $\mathrm{Eu}_{2} \mathrm{NiMnO}_{6}$ & $\mathrm{Gd}_{2} \mathrm{NiMnO}_{6}$ & $\mathrm{~Tb}_{2} \mathrm{NiMnO}_{6}$ \\
\hline Space Group & & $P 21 / n$ & $P 21 / n$ & $P 21 / n$ \\
\hline Cell Mass $(\mathrm{g} / \mathrm{mol})$ & & 1027.11 & 1048.26 & 1054.96 \\
\hline Cell Volume $\left(\AA^{3}\right)$ & & 222.86 & 221.56 & 219.58 \\
\hline Crystal Density $\left(\mathrm{g} / \mathrm{cm}^{3}\right)$ & 7.653 & 7.857 & 7.978 \\
\hline Lattice Parameters: & $a(\AA)$ & 5.3206 & 5.2915 & 5.2706 \\
\hline & $b(\AA)$ & 5.5251 & 5.4103 & 5.5358 \\
\hline & $c(\AA)$ & 7.5811 & 9.2220 & 7.5261 \\
\hline Rexp & $\beta\left({ }^{\circ}\right)$ & 90.003 & 124.976 & 90.158 \\
\hline Rwp & & 4.97 & 4.43 & 4.30 \\
\hline GoF & & 9.40 & 6.25 & 8.05 \\
\hline Bond length $(\AA):$ & $\mathrm{Ni}-0$ & 1.9961 & 2.0781 & 1.87 \\
\hline & $\mathrm{Mn}-0$ & 1.9481 & 1.9120 & 2.0444 \\
\hline Bond angle $\left({ }^{\circ}\right)$ & $\mathrm{Ni}-0-\mathrm{Mn}$ & 161.84 & 153.07 & 147.30 \\
\hline
\end{tabular}

The X-ray photoemission spectroscopy (XPS) technique is used to understand the chemical oxidation states and the ligand coordination of a system. The oxidation state analysis of manganese $(\mathrm{Mn})$ in ENMO, GNMO, and TNMO samples have been performed and Fig. $2(a, b, c)$ represents the XPS spectra for Mn2p. The spectra have been fitted with Shirley background subtraction. The deconvoluted XPS peak of $\mathrm{Mn} 2 \mathrm{p}_{3 / 2}$ splits into two peaks at the binding energy $641.2 \mathrm{eV}$ and $643.2 \mathrm{eV}$ which correspond to $\mathrm{Mn}^{4+}$ and $\mathrm{Mn}^{3+}$, respectively ${ }^{25}$. The ratio of $\mathrm{Mn}^{4+} / \mathrm{Mn}^{3+}$ for ENMO, GNMO, and TNMO is found to be $1.02,0.76$, and 1.64 respectively, indicating the presence of $\mathrm{Mn}^{4+}$ is more in the case of ENMO and TNMO while in the case of GNMO, $\mathrm{Mn}^{3+}$ is higher. The ratio of $\mathrm{Mn}^{4+} / \mathrm{Mn}^{3+}$ shows the change in surface oxidation state caused by different A-site rare earth elements having different ionic radii. This clearly indicate the superexchange mechanisms of $\mathrm{Ni}^{2+}-\mathrm{O}-\mathrm{Mn}^{3+}$ and $\mathrm{Ni}^{2+}-\mathrm{O}-\mathrm{Mn}^{4+}$ in the studied compounds are different.

Figure 3 (a) shows field-cooled (FC) temperature-dependent magnetization curves of ENMO, GNMO, and TNMO samples measured with an applied magnetic field of 100 Oe between 2-300 K. It is clearly seen that, with increasing the temperature, the magnetization in the samples decreased because of the magnetic phase transition temperature from ferromagnetic state to paramagnetic state. The studied samples show the same behavior which confirms the ferromagnetic to paramagnetic phase transition 
samples are shown in the inset to determine the Curie temperature (Tc). It is defined that the minima in the $\mathrm{dM} / \mathrm{dT}$ curve, and curie temperatures for ENMO, GNMO and TNMO samples are found to be 142, 130, and $112 \mathrm{~K}$, respectively. It is seen that with a decrease in rare earth size $\mathrm{Eu}^{3+}(0.947 \AA)>\mathrm{Gd}^{3+}(0.938 \AA)>$ $\mathrm{Tb}^{3+}(0.923 \AA)$, the $\mathrm{T}_{\mathrm{C}}$ shifts to lower temperature gradually which is probably due to the decreases of the $\mathrm{Ni}-\mathrm{O}-\mathrm{Mn}$ bond angle [161.84 (Eu), $153.07(\mathrm{Gd})$, and $147.30(\mathrm{~Tb})]^{26}$. The temperature dependent inverse susceptibility $\chi^{-1}(T)$ has been demonstrated in Fig. $3(b, c, d)$. The linear fitting of the experimental $\chi^{-1}(\mathrm{~T})$ curve with the Curie-Weiss (C-W) law $\chi=\frac{C}{T-\theta}$ in the paramagnetic region, where $\mathrm{C}$ is the Curie constant, and $\theta$ corresponds to paramagnetic Curie-Weiss temperature. The calculated values of effective magnetic are 8.6, 12.4, $15.1 \mu \mathrm{B} /$ f.u. for ENMO, GNMO, and TNMO, respectively, which are comparable with the corresponding theoretical values 7.0,12.2, $14.6 \mu \mathrm{B} / \mathrm{f}$.u. The difference in the theoretical and calculated effective magnetic moment is associated with the amount of $\mathrm{Mn}^{3+}$ and $\mathrm{Mn}^{4+}$ present in the compound. The positive values of Curie-Weiss temperature for studied DPs confirms the second-order magnetic phase transition. The Curie- Weiss fitting also shows that how evolution of Griffith phase with smaller ionic size of Eu, Gd, and Tb in double perovskite.

To investigate the magnetocaloric properties, the isothermal magnetization $(\mathrm{MH})$ curves at different temperatures were measured before and after the $T_{C}$. The temperature interval $\Delta T=3 \mathrm{~K}$ near to $T_{C}$, and $\Delta T=5 \mathrm{~K}$, rest of the region were kept constant. Figure $4(\mathrm{a}, \mathrm{b}, \mathrm{c})$ shows isothermal magnetization of ENMO, GNMO, and TNMO samples in a magnetic field range from $0-5 \mathrm{~T}$. The MH curves depicts that at lower magnetic field region, the $\mathrm{MH}$ curves rise rapidly and afterward with increasing magnetic field $\mathrm{MH}$ curves try to saturate, and this phenomenon is associated with ferromagnetic behavior of magnetic materials. The $\mathrm{MH}$ curves at higher temperatures region show linear behavior which confirms the paramagnetic nature of samples, and this is due to thermal agitation which disorients the magnetic moments at higher temperature. To know the order of magnetic phase transition, well-known Arrott plots $\left(\mathrm{M}^{2}\right.$ vs H/M) were analyzed, and which are deduced from the magnetic isotherms given in Fig. 4 (d, e, f). Banerjee's criterion, suggest that the slope of Arrott plots is important to know the type of magnetic phase transition. The negative slope corresponds to the first-order phase transition undergoes into the sample while positive slope confirms second-order phase transition ${ }^{27}$. It is seen that positive slope observed in the Arrott plot at all temperatures for studied samples. Therefore, we can confirm that ferromagneticparamagnetic transition is consistent of second-order type. The order of degree of the magnetic domains, variation of lattice volume and latent heat of phase transformation are extremely small in case of second order phase transition. It might be one of the reasons that the magnetic entropy changes of second-order phase transition materials smaller than that of first-order phase transition materials.

The magnetic entropy change determined by using the isothermal magnetization data, shown in Fig. 4, and which is initiated by the changing the applied magnetic field from 0 to $\mathrm{H}$ is determined by using the well-known Maxwell thermodynamic correlation, which is given by the equation, 


\section{$\Delta S_{M}(T, H)=\int_{0}^{H}\left(\frac{\partial M}{\partial T}\right)_{H} d H$}

Where, $\Delta \mathrm{S}$ is the entropy change, $\mathrm{dH}$ is the change in the applied magnetic field, $\mathrm{M}$ is the magnetization and $T$ is the temperature.

Figure $5(a, b, c)$ shows the temperature dependence of magnetic entropy curves $\left(-\Delta S_{M}\right)$ under different applied field changes ranging between 0 and $5 \mathrm{~T}$ for ENMO, GNMO, and TNMO. The maximum value of $\Delta S_{M}$ is found to be around the magnetic phase transition temperature. All these samples show similar behavior, $-\Delta S_{M}$ value reaches a maximum near the Curie temperature $\left(T_{C}\right)$ at low applied fields and it increases with increasing $\mathrm{H}$ which may be due to the improvement of FM interactions. The calculated $\Delta \mathrm{S}_{\mathrm{M}}$ are 3.2, 3.8, and 3.5 J/kgK at $\Delta \mathrm{H}=5 \mathrm{~T}$ for ENMO, GNMO, and TNMO samples, respectively. Rawat et al. The MCE properties of nanocrystalline $\mathrm{Pr}_{2} \mathrm{CoMnO}_{6}$ DPs prepared by sol-gel with an average particle size of $192 \mathrm{~nm}$, found to be $-\Delta S_{M}=1.98 \mathrm{~J} / \mathrm{kgK}$ at the field change of $5 \mathrm{~T}$ and relative cooling power (RCP) was calculated to be $110 \mathrm{~J} / \mathrm{kg}^{28}$. The MCE properties in $\mathrm{Eu}_{2} \mathrm{NiMnO}_{6}$ and $\mathrm{Dy}_{2} \mathrm{NiMnO}_{6}$ was reported by Su et al. The maximum value of magnetic entropy change $-\Delta \mathrm{S}_{\mathrm{M}}$ reaches $4.0 \mathrm{~J} / \mathrm{kg} \mathrm{K}$ for $\mathrm{Eu}_{2} \mathrm{NiMnO}_{6}$ and $5.2 \mathrm{~J} / \mathrm{kg} \mathrm{K}$ for $\mathrm{Dy}_{2} \mathrm{NiMnO}_{6}$ for a field change of 0-7 T, respectively ${ }^{23}$. Chakraborty et al. ${ }^{22}$ reported MCE value for $\mathrm{Ho}_{2} \mathrm{NiMnO}_{6}$ is $6.2 \mathrm{~J} / \mathrm{kg} \mathrm{K}$ and for $\mathrm{Tb}_{2} \mathrm{NiMnO}_{6}$ is $4.1 \mathrm{~J} / \mathrm{kg} \mathrm{K}$ with $\Delta \mathrm{H}=5 \mathrm{~T}$. From this study and literature, one interesting observation to be noted that as ionic radii of rare earth elements decrease in double perovskite there is the evolution of Griffith phase. From Fig. 3 (b, c, d) we can observe that inverse susceptibility does not obey the Curie-Weiss law for all samples, this is due to Griffith phase present in the GNMO and TNMO samples.

To know the effectiveness of MCE material, another an important parameter is measuring the cooling efficiency of the materials called relative cooling power (RCP). It is defined as, an amount of heat transferred between temperatures corresponding to the full width at half maximum of magnetic entropy curve, and it can be evaluated by following equation.

\section{$R C P=-\Delta S_{M} X \delta T_{F W H M}$}

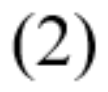

The RCP values are 150, 182, $176 \mathrm{~J} / \mathrm{kg}$ for ENMO, GNMO, and TNMO samples, respectively. The comparison of transition temperature, maximum entropy change, and RCP values have been summarized in Table 2 for the studied double perovskite and other reported double perovskite compounds. The $-\Delta S_{M}$ and RCP values for ENMO, GNMO, and TNMO does not show considerable change with different rare earth elements in double perovskite, change in Curie temperature $\left(T_{C}\right)$ observed. From Table 2, we can notice that the MCE parameters for studied DPs compounds are comparable with other DPs as well as other MCE materials, indicating ENMO, GNMO, and TNMO compounds are also considerable for magnetic cooling applications. The comparative study shows the Curie temperature and RCP behavior with decreasing ionic radii of the rare earth element in $\mathrm{Ln}_{2} \mathrm{NiMnO}_{6}$ double perovskite. Expecting a change in 
is not much feasible, because of phase transition in double perovskite is associated with $\mathrm{M}^{2+}-\mathrm{O}-\mathrm{Mn}^{4+}$ superexchange interaction but playing with working temperature this could be one of tool to tune the Curie temperature.

Table 2

Comparison of $-\triangle S_{M}$ and $R C P$ values for the $\mathrm{R}_{2} \mathrm{NiMnO}_{6}(\mathrm{R}$ $=\mathrm{Eu}, \mathrm{Gd}, \mathrm{Tb}, \mathrm{Dy}, \mathrm{Ho}, \mathrm{Er}$ ) sample.

\begin{tabular}{|llllll|}
\hline Compound & $\begin{array}{c}\mathrm{Tc} \\
(\mathrm{K})\end{array}$ & $\begin{array}{c}\Delta \mathrm{H} \\
(\mathrm{T})\end{array}$ & $\begin{array}{l}-\Delta \mathrm{S}_{\mathrm{M}} \\
(\mathrm{J} / \mathrm{kgK})\end{array}$ & $\begin{array}{l}\mathrm{RCP} \\
(\mathrm{J} / \mathrm{kg})\end{array}$ & Ref. \\
\hline $\mathrm{Eu}_{2} \mathrm{NiMnO}_{6}$ & 143 & 5 & 3.2 & 150 & Present \\
\hline $\mathrm{Gd}_{2} \mathrm{NiMnO}_{6}$ & 130 & 5 & 3.7 & 182 & Present \\
\hline $\mathrm{Tb}_{2} \mathrm{NiMnO}_{6}$ & 112 & 5 & 3.5 & 176 & Present \\
\hline $\mathrm{Dy}_{2} \mathrm{NiMnO}_{6}$ & 101 & 5 & 3.4 & 175 & 19 \\
\hline $\mathrm{Ho}_{2} \mathrm{NiMnO}_{6}$ & 93 & 5 & 3.7 & 194 & 19 \\
\hline $\mathrm{Er}_{2} \mathrm{NiMnO}_{6}$ & 84 & 5 & 3.4 & 169 & 19 \\
\hline
\end{tabular}

\section{Conclusions}

In summary, we have synthesized $\mathrm{Eu}_{2} \mathrm{NiMnO}_{6}$ (ENMO), $\mathrm{Gd}_{2} \mathrm{NiMnO}_{6}$ (GNMO) and $\mathrm{Tb}_{2} \mathrm{NiMnO}_{6}$ (TNMO) double perovskite by using solid state reaction, where we found that all the samples are confirmed to the monoclinic structure with $P 2{ }_{1} / n$ space group. XRD, XPS, MT, and MCE data have been systematically investigated. All the samples exhibit a second-order magnetic phase transition, with the curie temperature $\left(T_{C}\right) 142,130$ and $112 \mathrm{~K}$, respectively for ENMO, GNMO, and TNMO samples. The double perovskite structure, which is associated with the superexchange mechanism, the decrease in Curie temperature is due to a decrease in ionic radii of Eu, Gd, and Tb. The $-\Delta S_{M}$ and RCP at an applied field of $5 \mathrm{~T}$ are found to be $3.2,3.8 \mathrm{~J} / \mathrm{kgK}, 3.5 \mathrm{~J} / \mathrm{kgK}$ and $150,182,176 \mathrm{~J} / \mathrm{kgK}$ respectively for the studied sample. The $-\Delta \mathrm{S}_{M}$ and RCP indicate the applicability of studied compound $\mathrm{Eu}_{2} \mathrm{NiMnO}_{6}, \mathrm{Gd}_{2} \mathrm{NiMnO}_{6}$, and $\mathrm{Tb}_{2} \mathrm{NiMnO}_{6}$ as magnetic refrigerants working in a wide temperature range, they are very promising magnetic refrigerant materials. We propose that the double perovskite with $A$ - site with rare earth element and $B-$ site with $3 \mathrm{~d}$ ferromagnetic elements $\mathrm{Fe}, \mathrm{Co}$, and $\mathrm{Ni}$ with $\mathrm{Mn}$ can be reliable and systematic playground in engineering magnetocaloric properties for future applications.

\section{Experimental}


Polycrystalline double perovskite compounds of $\mathrm{Eu}_{2} \mathrm{NiMnO}_{6}$ (ENMO), $\mathrm{Gd}_{2} \mathrm{NiMnO}_{6}(\mathrm{GNMO}), \mathrm{Tb}_{2} \mathrm{NiMnO}_{6}$ (TNMO) in the present study were synthesized by conventional solid-state synthesis. The precursors $\mathrm{Eu}_{2} \mathrm{O}_{3}, \mathrm{Gd}_{2} \mathrm{O}_{3}, \mathrm{~Tb}_{2} \mathrm{O}_{3}, \mathrm{NiO}$, and $\mathrm{MnO}_{2}$ weighted in stoichiometric amounts were mixed and grinded in the mortar and were heat-treated initially at $900^{\circ} \mathrm{C}$ for $24 \mathrm{~h}$, after regrinding heat-treated at $1100^{\circ} \mathrm{C}$ for $24 \mathrm{~h}$, and finally all samples sintered at $1300^{\circ} \mathrm{C}$ for $48 \mathrm{~h}$ after regrinding. The phase formation of sintered ceramic compounds was checked by X-ray diffraction (XRD) by using the X-ray diffractometer (Rigaku) and analyzed by Rietveld method by using TOPAS software. The X-ray photoelectron spectroscopy (XPS, VersaProbe, PHI 5000) measurement was carried out by using an Al-Ka radiation source under $15 \mathrm{kV}$ voltage and $5 \mathrm{~mA}$ current. The XPS data was analyzed by using the XPSpeak41 software. The temperature dependent magnetization and isothermal magnetization of the samples were measured in a vibrating sample magnetometer (VSM) based on Superconducting Quantum Interference Device (SQUID) from Quantum Design.

\section{Declarations}

\section{Acknowledgements}

This study was supported by the Science Research Program through the National Research Foundation in Republic of Korea (NRF) funded by the Ministry of Education, Science and Technology (NRF contract No. 2020R1I1A3070554). S. Park and Y. Jo acknowledge the support by the KBSI grant (D110200).

\section{Author contributions}

K. P. Shinde and E. J. Lee performed experiments and analyzed data. M. Manawan contributed to Rietveld's analysis of X-ray diffraction. A. Lee, S.Y. Park, and Y. Jo contributed to the magnetic measurements by MPMS. K. Ku and J.M. Kim contributed with scientific discussion on the manuscript. J. S. Park planned the study and K.P. Shinde wrote the manuscript with the input from all collaborators. All authors discussed the results and reviewed the manuscript.

\section{Competing financial interests:}

The authors declare no competing financial interests.

\section{References}

1. Gschneidner, K. A. Jr., Pecharsky, V. K. \& Tsokol, A. O. Recent developments in magnetocaloric materials. Rep. Prog. Phys. 68, 1479, https://doi.org/10.1088/0034-4885/68/6/R04 (2005).

2. Yu, B.F., Gao, Q., Zhang, B., Meng, X. Z. \& Chen, Z. Review on research of room temperature magnetic refriaeration. Int. J. Refria. 26. 622. https://doi.ora/10.1016/S0140- 7007(03)00048-3 (2003). 
3. Brück, E. Developments in magnetocaloric refrigeration. J. Phys. D Appl. Phys. 38, R381, https://doi.org/10.1088/0022-3727/38/23/R01 (2005).

4. Gschneidner, K. A. \& Pecharsky, V. K. Rare earths, and magnetic refrigeration. J. Rare Earth 24, 641, https://doi.org/10.1016/S1002-0721(07)60001-5 (2006).

5. Tishin, A. M. \& Spichkin, Y. I. The Magnetocaloric Effect and Its Applications. Institute of Physics, Bristol. https://doi.org/10.1201/9781420033373 (2003)

6. Benford, S. M. \& Brown, G. V. T-S diagram for gadolinium near the curie temperature. J. Appl. Phys. 52, 2110, https://doi.org/10.1063/1.329633 (1981).

7. Phan, M. H. \& Yu, S. C. Review of the magnetocaloric effect in manganites materials. J. Magn. Magn. Mater. 308, 325, https://doi.org/10.1016/j.jmmm.2006.07.025 (2007).

8. Meng, J., Liu, X., Hao, X., Zhang, L., Jian, F. Y. \& Zhang, H. Microscopic mechanistic study on the multiferroic of $\mathrm{R}_{2} \mathrm{CoMnO}_{6} / \mathrm{La}_{2} \mathrm{CoMnO}_{6}(\mathrm{R}=\mathrm{Ce}, \mathrm{Pr}, \mathrm{Nd}, \mathrm{Pm}, \mathrm{Sm}, \mathrm{Gd}, \mathrm{Tb}, \mathrm{Dy}, \mathrm{Ho}, \mathrm{Er}, \mathrm{Tm})$ by chemical and hydrostatic pressures: a first-principles calculation. J. Phys. Chem. Chem. Phys. 18, 23613, https://doi.org/10.1039/C6CP03145E (2016).

9. Kumar, A. \& Mahato, R. N. Effect of annealing temperature on structural and magnetocaloric properties of nanocrystalline $\mathrm{BaCo}_{0.90} \mathrm{Mn}_{0.10} \mathrm{O}_{3-\delta}$. J. Alloys Compd. 723,

675, https://doi.org/10.1016/j.jallcom.2017.06.267 (2017).

10. Gomes, A. M., Garcia, F., Guimara, A. P., Reis, M. S., Amaral, V. S. \& Tavares, P. B. Magnetocaloric effect of the $(\mathrm{Pr}, \mathrm{Ca}) \mathrm{MnO}_{3}$ manganite at low temperatures. J. Magn. Magn. Mater. 290, 694, https://doi.org/10.1016/j.jmmm.2004.11.339 (2005).

11. Shimakawa, Y., Azuma, M. \& Ichikawa, N. Multiferroic Compounds with Double-Perovskite Structures. Materials. 4, 153, https://doi.org/10.3390/ma4010153 (2011).

12. Mahato, R. N., Sethupathi, K. \& Sankaranarayanan, V. Colossal magnetoresistance in the double perovskite oxide $\mathrm{La}_{2} \mathrm{CoMnO}_{6}$. J. Appl. Phys. 107, 09D714, https://doi.org/10.1063/1.3350907 (2010).

13. Yang, D., Wang, W., Yang, T., Lampronti, G. I., Ye, H., Wu, L., Yu, Q. \& Lu, S. Role of spontaneous strains on the biphasic nature of partial B-site disorder double perovskite $\mathrm{La}_{2} \mathrm{NiMnO}_{6}$. APL Materials 6, 066102, https://doi.org/10.1063/1.5031486 (2018).

14. Kimura, T., Goto, T., Shintani, H., Ishizaka, K., Arima, T. \& Tokura, Y. Magnetic control of ferroelectric polarization. Nature 426, 55, https://doi.org/10.1038/nature02018 (2003).

15. Rogado, N. S., Li, J., Sleight, A. W. \& Subramanian, M. A. Magnetocapacitance and Magnetoresistance Near Room Temperature in a Ferromagnetic Semiconductor: $\mathrm{La}_{2} \mathrm{NiMnO}_{6}$. Adv. Mater. 17, 2225, https://doi.org/10.1002/adma.200500737 (2005).

16. Murthy, J. K., Chandrasekhar, K. D., Mahana, S., Topwal, D. \& Venimadhav, A. Giant magnetocaloric effect in $\mathrm{Gd}_{2} \mathrm{NiMnO}_{6}$ and $\mathrm{Gd}_{2} \mathrm{CoMnO}_{6}$ ferromagnetic Insulators. J. Phys. D: Appl. Phys. 48, 355001, https://doi.org/10.1088/0022-3727/48/35/355001 (2015). 
17. Zhang, Y., Li, H., Guo, D., Hou, L., Li, X., Ren, Z. \& Wilde, G. Cryogenic magnetic Properties and magnetocaloric performance in double perovskite $\mathrm{Pr}_{2} \mathrm{NiMnO}_{6}$ and $\mathrm{Pr}_{2} \mathrm{CoMnO}_{6}$ compounds. Ceram. Int. 44, 20762, https://doi.org/10.1016/j.ceramint.2018.08.075 (2018).

18. Moon, J. Y., Kim, M. K., Oh, D. G., Kim, J. H., Shin, H. J., Choi, Y. J. \& Lee, N. Anisotropic magnetic properties and giant rotating magnetocaloric effect in double perovskite $\mathrm{Tb}_{2} \mathrm{CoMnO}_{6}$. Phys. Rev. $\mathrm{B}$ 98, 174424. https://doi.org/10.1103/PhysRevB.98.174424 (2018).

19. Jia, Y., Wang, Q., Qi, Y. \& Li, L. Multiple magnetic phase transitions and magnetocaloric effect in double perovskites $R_{2} \mathrm{NiMnO}_{6}(R=\mathrm{Dy}, \mathrm{Ho}$, and Er). J. Alloy. Compd. 726, 1132, https://doi.org/10.1016/j.jallcom.2017.08.073 (2017).

20. Balli, M., Fournier, P., Jandl, S. \& Gospodinov, M. M. A study of the phase transition and magnetocaloric effect in multiferroic $\mathrm{La}_{2} \mathrm{MnNiO}_{6}$ single crystals. J. Appl. Phys. 115, 173904, https://doi.org/10.1063/1.4874943 (2014).

21. Balli, M., Fournier, P., Jindal, S., Truong, K. D. \& Gospodinov, M. M. Analysis of the phase transition and magneto-thermal properties in $\mathrm{La}_{2} \mathrm{CoMnO}_{6}$ single crystals. J. Appl. Phys. 116, 073907, https://doi.org/10.1063/1.4893721 (2014).

22. Chakraborty, T., Nhalil, H., Yadav, R., Wagh, A. A. \& Elizabeth, S. Magnetocaloric properties of $\mathrm{R}_{2} \mathrm{NiMnO}_{6}(\mathrm{R}=\mathrm{Pr}, \mathrm{Nd}, \mathrm{Tb}, \mathrm{Ho}$ and $\mathrm{Y})$ double perovskite family. J. Magn. Magn. Mater. 428, 59, https://doi.org/10.1016/j.jmmm.2016.12.015 (2017).

23. Su, L., Zhang, X. Q., Dong, Q. Y., Ke, Y.J., Hou, K.Y., Liu, C. S. \& Cheng, Z. H. Magnetocaloric effect and critical behaviors of $\mathrm{R}_{2} \mathrm{NiMnO}_{6}(\mathrm{R}=\mathrm{Eu}$ and $\mathrm{Dy}$ ) double perovskite oxides. J. Alloys Compd. 746, 594, https://doi.org/10.1016/j.jallcom.2018.02.327 (2018).

24. Coelho, A. A. TOPAS and TOPAS-Academic: an optimization program integrating computer algebra and crystallographic objects written in $\mathrm{C}^{++}$. J. Appl. Cryst. 51, 210, https://doi.org/10.1107/S1600576718000183 (2018).

25. Wang, Q., Ma, L., Wang, L. \& Wang, D. The superior NO oxidation activity over $\mathrm{La}_{2} \mathrm{CoMnO}_{6}$ double perovskite to that of $\mathrm{La}_{2} \mathrm{GaMnO}_{6}$ : effects of magnetic $\mathrm{Co}^{3+}$ and nonmagnetic $\mathrm{Ga}^{3+}$. New $\mathrm{J} \mathrm{Chem.} \mathrm{43,}$ 13911, https://doi.org/10.1039/C9NJ03248G (2019).

26. Kakarla, D. C., Jyothinagaram, K. M., Das, A. K. \& Adyam, V. Dielectric and Magnetodielectric Properties of $\mathrm{R}_{2} \mathrm{NiMnO}_{6}(\mathrm{R}=\mathrm{Nd}$, Eu, Gd, Dy, and $\mathrm{Y})$. J. Am. Ceram. Soc. 97, 2858, https://doi.org/10.1111/jace.13039 (2014).

27. Banerjee, S. K. On a generalised approach to first and second order magnetic transitions. Phys. Lett. 12, 16, https://doi.org/10.1016/0031-9163(64) 91158-8 (1964).

28. Rawat, K., Meenakshi \& Mahato, R. N. Investigation of structural, magnetic, and magnetocaloric properties of nanocrystalline double perovskite $\mathrm{Pr}_{2} \mathrm{CoMnO}_{6}$. Mater. Res. Express 5, 066110, https://doi.org/10.1088/2053-1591/aacd92 (2018). 
(a)

(b)
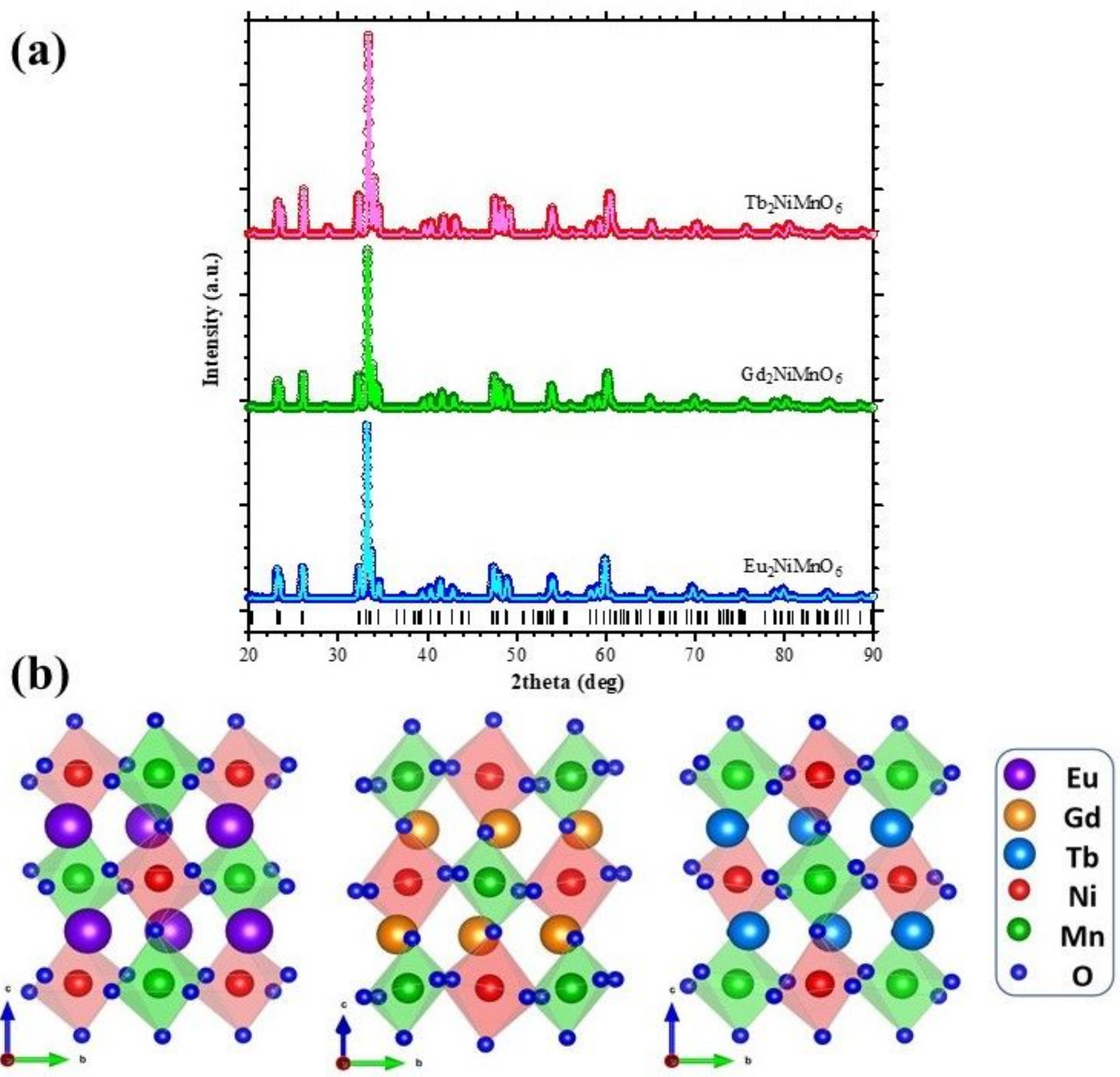

Figure 1

Rietveld refinement of x-ray diffraction data and crystal structure of ENMO, GNMO, and TNMO samples. In crystallographic structure blue, green, olive, pink, gray, and red spheres represent Eu3+, Gd3+, Tb3+, $\mathrm{Ni2+,} \mathrm{Mn4+,} \mathrm{and} \mathrm{02-} \mathrm{ions,} \mathrm{respectively.}$ 

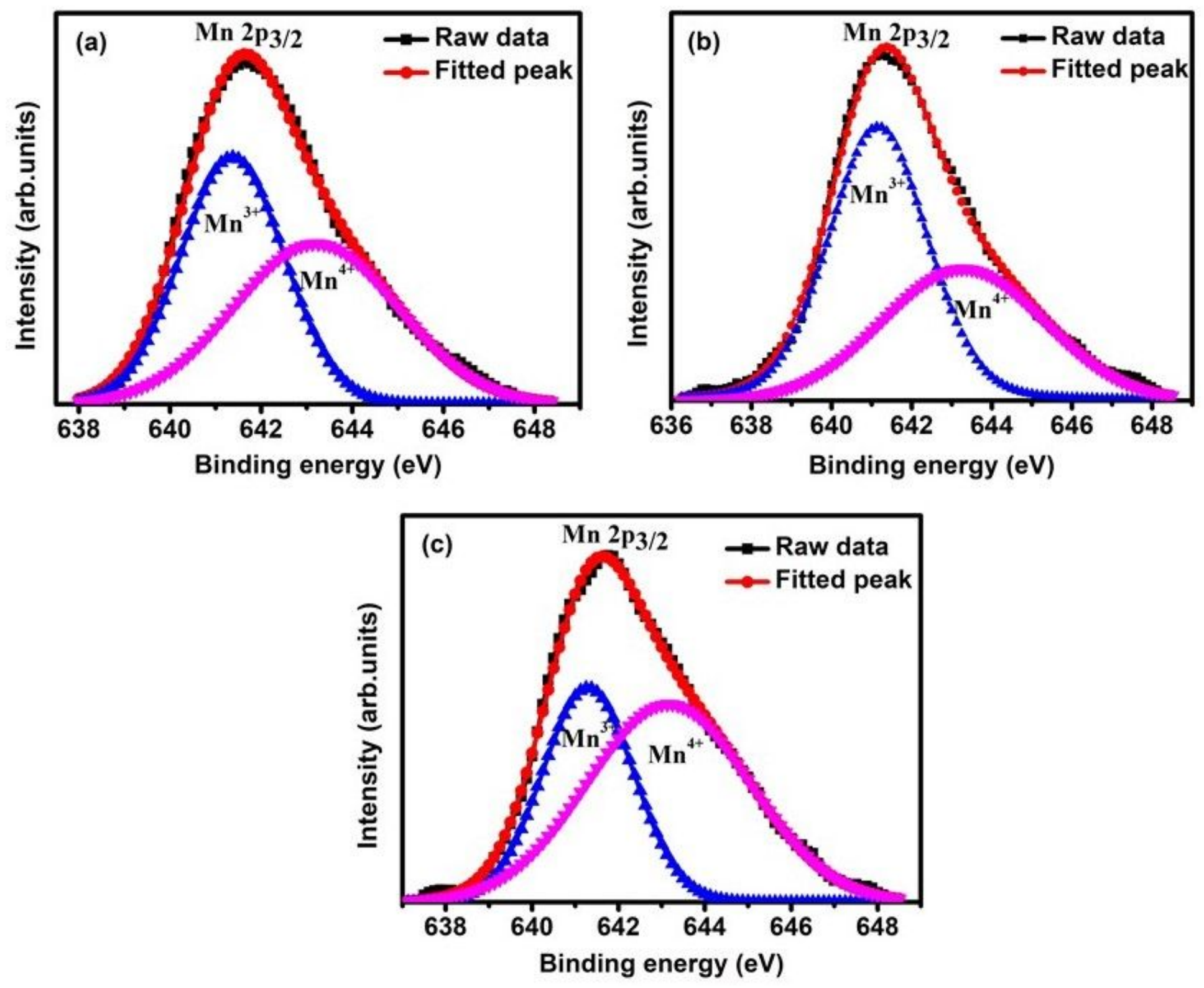

Figure 2

High-resolution XPS spectra of Mn2p for (a) ENMO (b) GNMO (c) TNMO samples. 

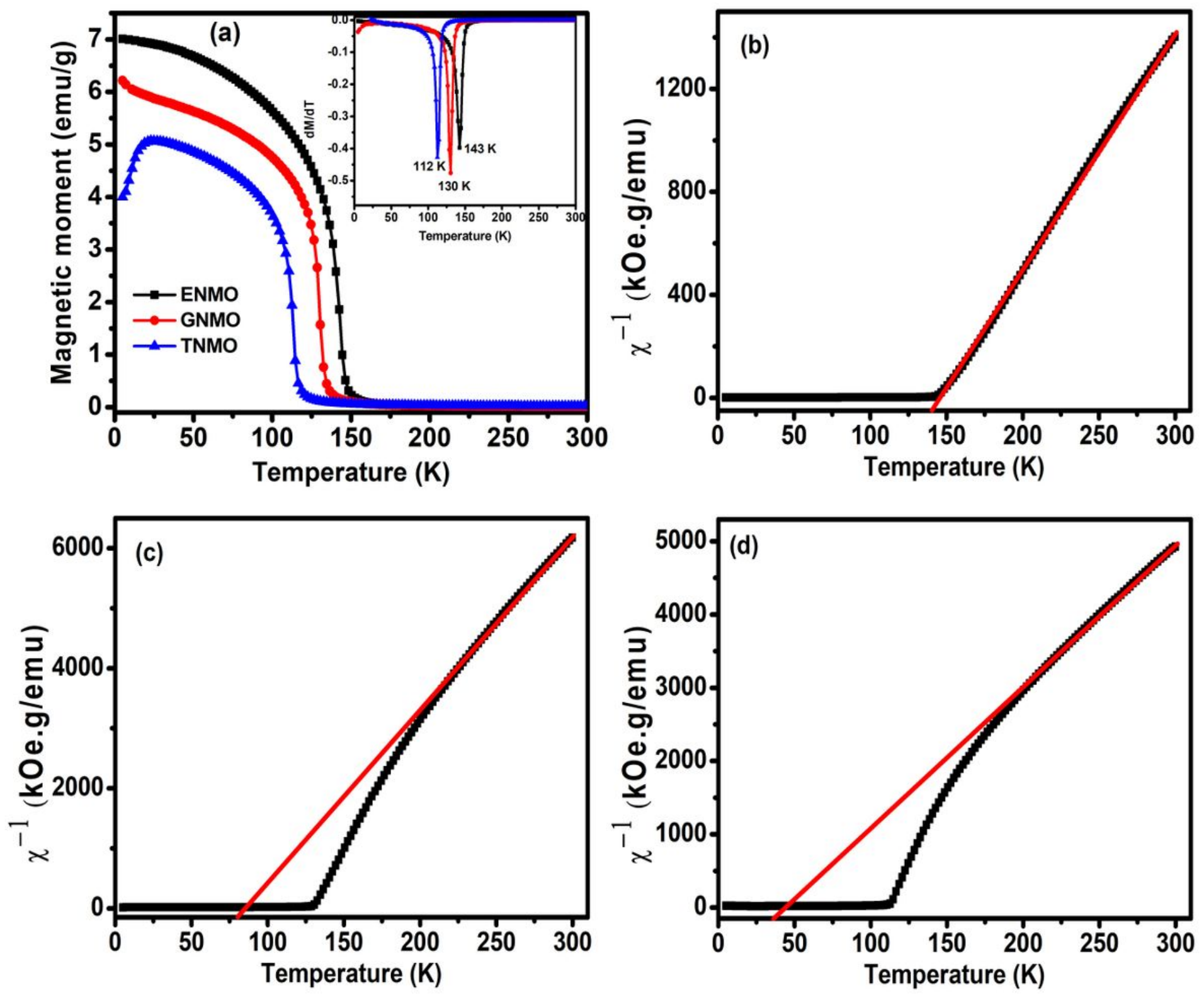

Figure 3

(a) The temperature dependent magnetization under the magnetic field of $100 \mathrm{Oe}$. Inset shows variation of $\mathrm{dM} / \mathrm{dT}$ curve with temperature. (b, c, d) Temperature dependence of the inverse magnetic susceptibility and Curie-Weiss fitting for ENMO, GNMO and TNMO samples. 

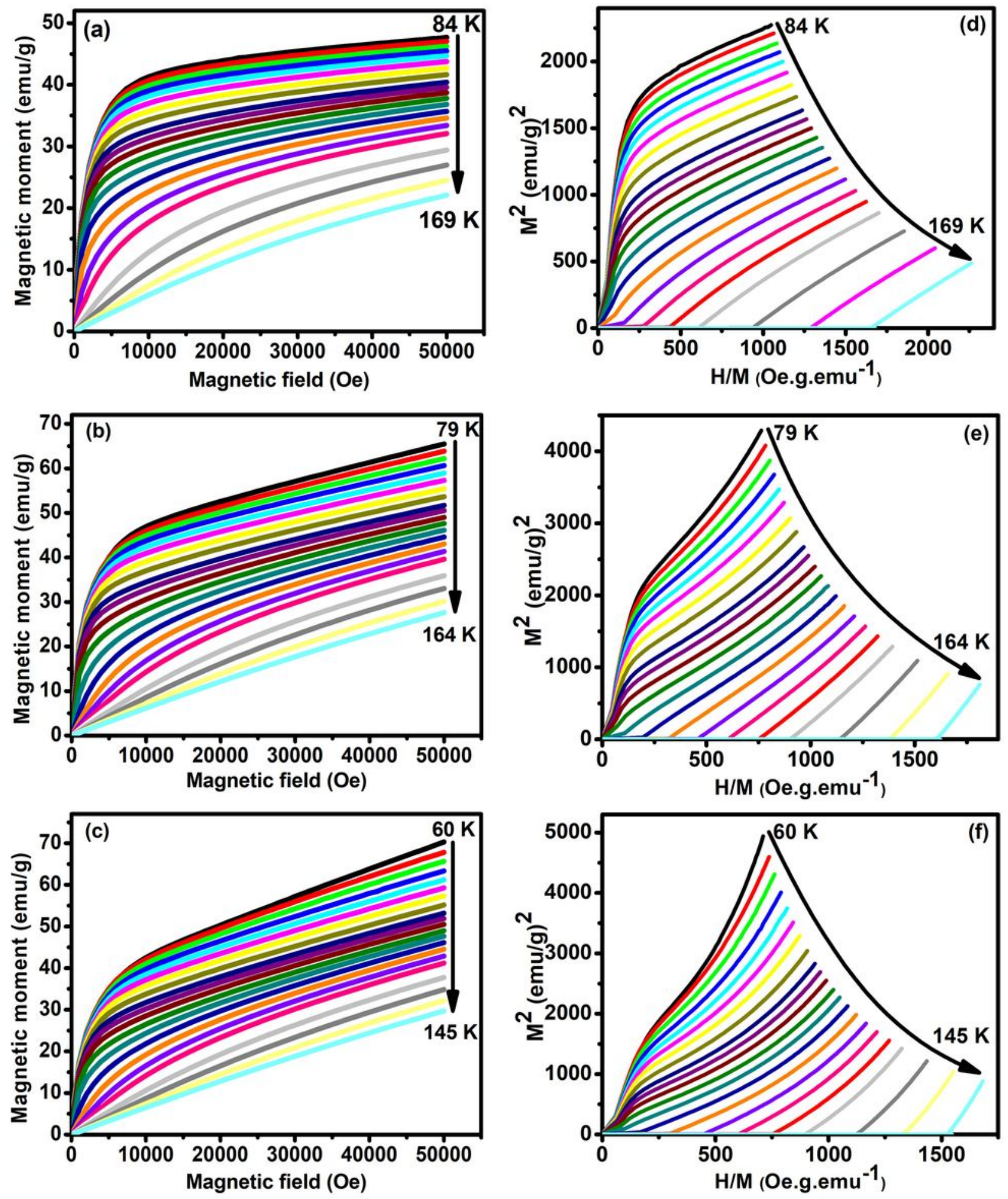

Figure 4

$(a, b, c)$ Isothermal magnetization curves $(\mathrm{MH})$ around TC (d, e, f) Arrott plot for ENMO, GNMO and TNMO samples. 

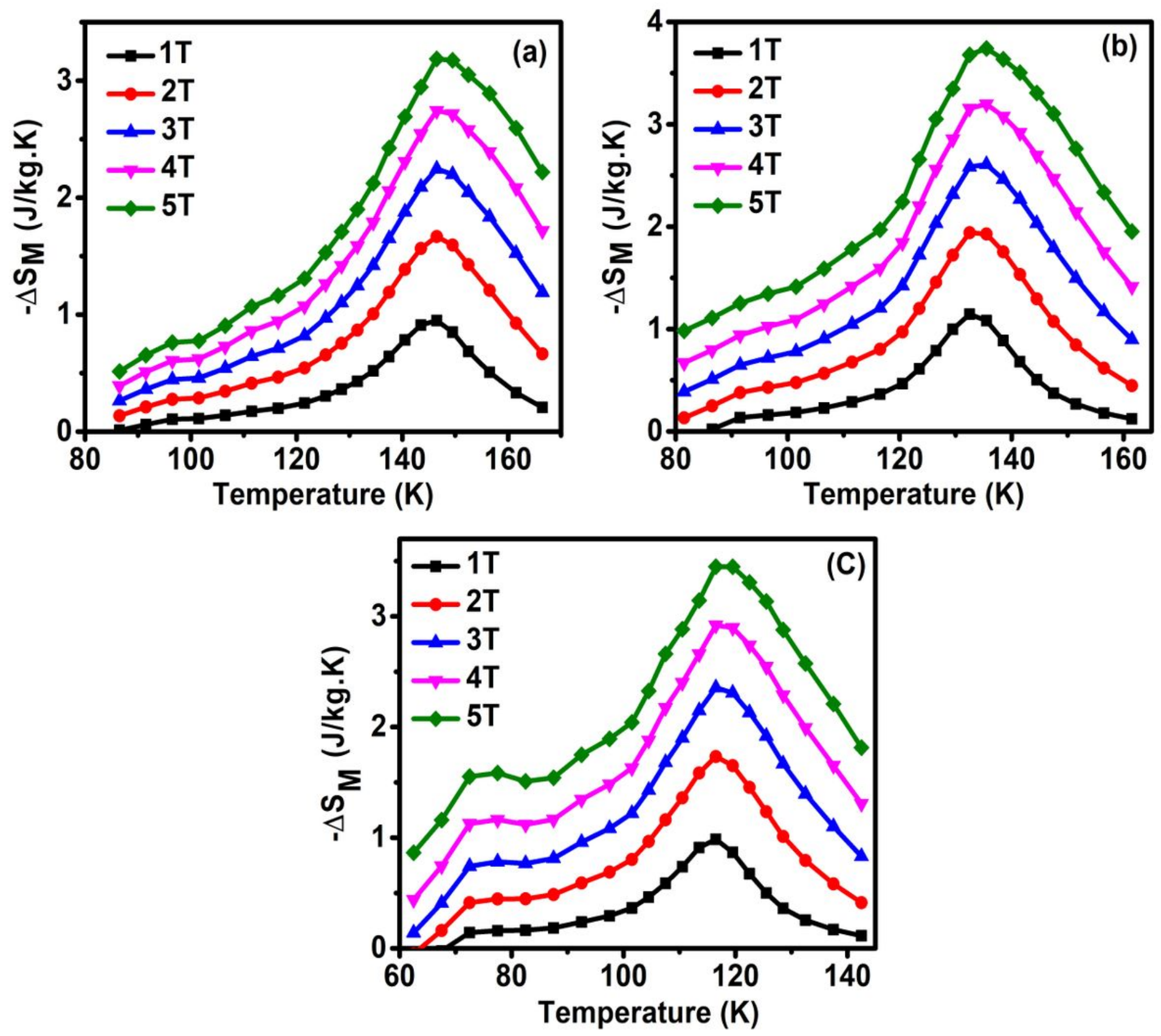

Figure 5

Magnetic entropy change $(-\Delta S M)$ with respect of temperature in the magnetic field $1-5 \mathrm{~T}$ for (a) ENMO, (b) GNMO and (c) TNMO samples. 